University of Nebraska - Lincoln

DigitalCommons@University of Nebraska - Lincoln

Matthias Fuchs Publications

Research Papers in Physics and Astronomy

2010

First Milestone on the path toward a table-top free-electron laser (FEL)

Matthias Fuchs

University of Nebraska-Lincoln, mfuchs@unl.edu

R. Weingartner

Max-Planck-Institut für Quantenoptik

A. Popp

Max-Planck-Institut für Quantenoptik

Zs. Major

Max-Planck-Institut für Quantenoptik

S. Becker

Max-Planck-Institut für Quantenoptik

See next page for additional authors

Follow this and additional works at: https://digitalcommons.unl.edu/physicsfuchs

Fuchs, Matthias; Weingartner, R.; Popp, A.; Major, Zs.; Becker, S.; Osterhoff, J.; Seggebrock, T.; Horlein, R.; Tsakiris, G.D.; Schramm, U.; Rowlands-Ree, T.P.; Hooker, S.M.; Habs, D.; Krausz, F.; Karsch, S.; and Gruner, F., "First Milestone on the path toward a table-top free-electron laser (FEL)" (2010). Matthias Fuchs Publications. 1.

https://digitalcommons.unl.edu/physicsfuchs/1

This Article is brought to you for free and open access by the Research Papers in Physics and Astronomy at DigitalCommons@University of Nebraska - Lincoln. It has been accepted for inclusion in Matthias Fuchs Publications by an authorized administrator of DigitalCommons@University of Nebraska - Lincoln. 


\section{Authors}

Matthias Fuchs, R. Weingartner, A. Popp, Zs. Major, S. Becker, J. Osterhoff, T. Seggebrock, R. Horlein, G.D. Tsakiris, U. Schramm, T.P. Rowlands-Ree, S.M. Hooker, D. Habs, F. Krausz, S. Karsch, and F. Gruner 


\section{AIP Conference Proceedings}

First milestone on the path toward a tabletop freeelectron laser (FEL)

M. Fuchs, R. Weingartner, A. Popp, Zs. Major, S. Becker et al.

Citation: AIP Conf. Proc. 1228, 295 (2010); doi: 10.1063/1.3426066

View online: http://dx.doi.org/10.1063/1.3426066

View Table of Contents: http://proceedings.aip.org/dbt/dbt.jsp?KEY=APCPCS\&Volume=1228\&lssue=1

Published by the American Institute of Physics.

\section{Additional information on AIP Conf. Proc.}

Journal Homepage: http://proceedings.aip.org/

Journal Information: http://proceedings.aip.org/about/about_the_proceedings

Top downloads: http://proceedings.aip.org/dbt/most_downloaded.jsp?KEY=APCPCS

Information for Authors: http://proceedings.aip.org/authors/information_for_authors

- Article-level metrics now available 


\title{
First milestone on the path toward a table-top free-electron laser (FEL)
}

\author{
M. Fuchs ${ }^{*, \dagger}$, R. Weingartner, ${ }^{* \dagger}$, A. Popp ${ }^{*}$, Zs. Major*, S. Becker, ${ }^{*, \dagger}$ J. \\ Osterhoff*, ${ }^{*}$, T. Seggebrock ${ }^{\dagger}$, R. Hörlein ${ }^{*}$, , G.D. Tsakiris*, U. Schramm**, \\ T.P. Rowlands-Rees ${ }^{\ddagger}$, S.M. Hooker ${ }^{\ddagger}$, D. Habs ${ }^{*, \dagger}$, F. Krausz ${ }^{*, \dagger}$, S. Karsch ${ }^{*, \dagger}$ \\ and F. Grüner*, ${ }^{*}$ \\ ${ }^{*}$ Max-Planck-Institut für Quantenoptik, Hans-Kopfermann-Str. 1, 85748 Garching, Germany \\ ${ }^{\dagger}$ Dept. of Physics, Ludwig-Maximilians-Universität München, Am Coulombwall 1, 85748 \\ Garching, Germany \\ ${ }^{* *}$ Forschungszentrum Dresden-Rossendorf, Bautzner Landstrasse 128, 01328 Dresden, Germany \\ ${ }^{\sharp}$ University of Oxford, Clarendon Laboratory, Parks Road, Oxford OXI 3PU, U.K.
}

\begin{abstract}
Latest developments in the field of laser-wakefield accelerators (LWFAs) have led to relatively stable electron beams in terms of peak energy, charge, pointing and divergence from $\mathrm{mm}$ sized accelerators. Simulations and LWFA theory indicate that these beams have low transverse emittances and ultrashort bunch durations on the order of $\sim 10 \mathrm{fs}$. These features make LWFAs perfectly suitable for driving high-brightness X-ray undulator sources and free-electron lasers (FELs) on a university-laboratory scale. With the detection of soft-X-ray radiation from an undulator source driven by laser-wakefield accelerated electrons, we succeeded in achieving a first milestone on this path. The source delivers remarkably stable photon beams which is mainly due to the stable electron beam and our miniature magnetic quadrupole lenses, which significantly reduce its divergence and angular shot-to-shot variation. An increase in electron energy allows for compact, tunable, hard-Xray undulator sources. Improvements of the electron beams in terms of charge and energy spread will put table-top FELs within reach.
\end{abstract}

Keywords: Laser-plasma acceleration, Undulator radiation, Table-top FEL

PACS: $52.38 . \mathrm{Kd}, 41.60 . \mathrm{Ap}, 41.60 . \mathrm{Cr}$

\section{INTRODUCTION}

Synchrotrons and free-electron lasers are the most powerful sources that can produce brilliant and tunable X-ray radiation. They have become invaluable tools for research in a broad range of fields in science and technology [1]. Both of these sources consist of an electron accelerator and an undulator, which is a periodic magnetic structure that forces the electrons to oscillate and emit radiation. However, due to the large size of radiofrequency-based particle accelerators, the dimensions of these facilities are usually on the kilometer scale, which limits their number to only a few installations worldwide.

Novel particle accelerators that are driven by ultrahigh-intensity femtosecond laser pulses are able to accelerate electron bunches to quasi-monoenergetic distributions in the $\mathrm{GeV}$ range over distances of only a few $\mathrm{mm}[2,3,4]$, which is more than three orders of magnitude smaller than in their conventional counterparts. Besides the small dimensions of the accelerator, LWFA theory and simulations $[5,6]$ predict the electron bunches to be ultrashort with durations on the order of $10 \mathrm{fs}$ and of low transverse emittance. 
This makes them perfectly suitable for driving highly brilliant X-ray undulator sources that are affordable and maintainable by university-scale laboratories and therefore could markedly increase the accessibility of such sources for many users.

The concept of laser-wakefield accelerators is based on a few-femtosecond laser pulse with intensities in the terawatt to petawatt scale that is focussed into a gas target. The laser ionizes the atoms of the gas and excites a plasma wave, which trails the laser pulse at its group velocity. The electric fields generated by the wave are called wakefields and can be more than three orders of magnitude larger than in conventional accelerators. If the laser intensity is high enough, the plasma wave breaks and electrons can become trapped and "surf" these accelerating fields. This allows them to reach relativistic energies within distances of only a few millimeters. LWFA simulations and theory predict that the pulse duration of these electron beams is smaller than half of the plasma wavelength (typically a few tens of $\mu \mathrm{m}$ ) and therefore is estimated to be on the order of $10 \mathrm{fs}$. The fields are also expected to sustain electron bunches with charges of several hundred $\mathrm{pC}$. The highest experimentally verified value is $300 \mathrm{pC}$ [2]. This large amount of charge in ultrashort electron bunches result in extremely high peak currents, a key prerequisite for the realization of table-top FELs [7].

After exiting the plasma, the electron beam is collimated by a pair of miniature magnetic quadrupole lenses and propagates through the undulator, where it emits radiation. An undulator is a periodic magnetic structure that forces the electrons on a sinusoidal trajectory whereby they oscillate transversely to their direction of propagation. Such an acceleration causes them to emit spontaneous radiation with a wavelength of

$$
\lambda=\frac{\lambda_{u}}{2 n \gamma^{2}}\left(1+\frac{K^{2}}{2}+\gamma^{2} \Theta^{2}\right),
$$

where $n$ is the number of the emitted harmonic, $\gamma$ is the total electron energy in terms of the electron rest mass $E=m_{0} c^{2}, \Theta$ is the angle of the emitted radiation with respect to the direction of propagation of the electron. The deflection parameter $K \propto B_{0} \cdot \lambda_{u}$ is proportional to the product of the on-axis undulator magnetic field $B_{0}$ and its period $\lambda_{u}$.

The radiation emitted in spontaneous undulator sources is incoherent, whereas FELs emit coherently. The coherent FEL emission is a result of the so-called microbunching of the electron bunch. This is a consequence of the spontaneous undulator radiation acting back on the electrons in the bunch, accelerating and decelerating some of them. Eventually, this results in a periodic density modulation (the microbunches) that are separated by a wavelength of the emitted radiation. The distance between two electrons within these microbunches is smaller than the wavelength that they are emitting and therefore the emission is coherent. This means that the emitted intensity is proportional to $N_{e}^{2}$ ( $N_{e}$ is the number of electrons in the bunch), whereas the spontaneous radiation scales only with $N_{e}$. The FEL process requires a high quality electron beam in terms of $3 \mathrm{D}$ charge density, energy spread and emittance. The concept of table-top FELs [7, 8] is based on laser-wakefield accelerated bunches with a high amount of charge in a short pulse duration, corresponding to a high peak current. This leads to a much shorter FEL saturation length, which allows for a significant reduction in the length of the undulator compared to FELs driven by conventional accelerators. 


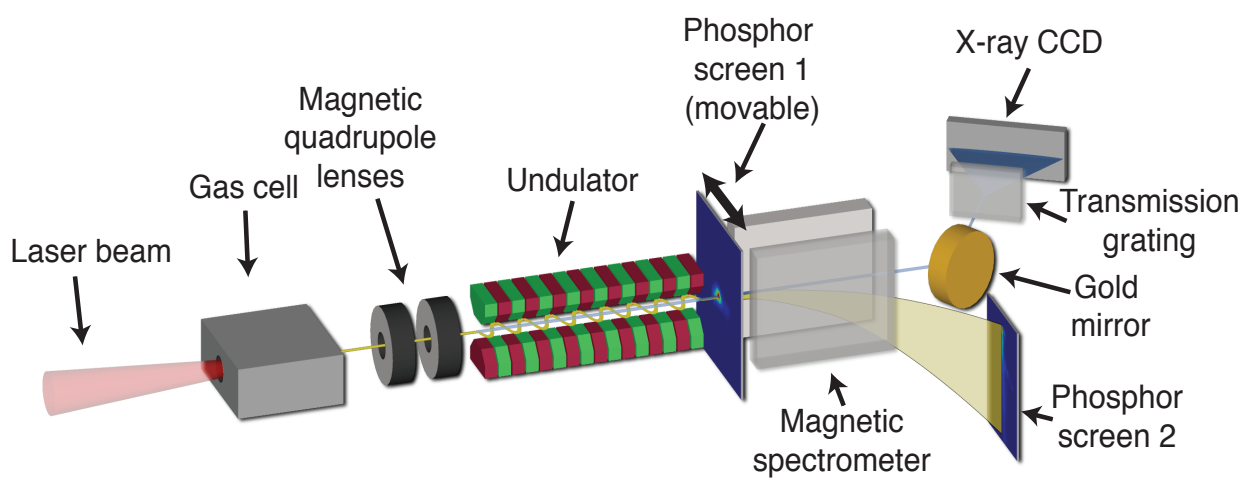

FIGURE 1. Experimental Setup. A laser pulse (red) is focused into a gas target, where it accelerates electrons (yellow). The laser and plasma radiation are blocked by a thin aluminum foil. Subsequently, the electron beam is collimated by a pair of magnetic quadrupole lenses and propagates through the undulator, where it emits undulator radiation (blue). The radiation is characterized by an X-ray spectrometer, consisting of a transmission grating and an X-ray CCD. The pointing and divergence of the electron beam can be diagnosed by a movable phosphor screen. Simultaneous to the undulator spectrum, the electron spectrum can be measured by a second phosphor screen located after a dipole magnet.

\section{EXPERIMENTAL SETUP}

The wakefield accelerator is driven by the 20 TW Ti:Sapphire ATLAS laser system, which delivers pulses with an energy of $850 \mathrm{~mJ}$ on target and a $37 \mathrm{fs}$ duration at a central wavelength of $800 \mathrm{~nm}$ and a repetition rate of $10 \mathrm{~Hz}$. The pulses are focussed into the gas target by an $\mathrm{f} / 22$ off-axis parabola onto a $23 \mu \mathrm{m}$ full-width at half-maximum (FWHM) focal spot, which corresponds to a normalized laser vector potential of $a_{0} \simeq 1$. The target is a hydrogen-filled gas cell with a diameter of $\sim 200 \mu \mathrm{m}$ and a length of $15 \mathrm{~mm}$. The laser ionizes the hydrogen and produces a plasma. At a plasma density of $n_{p} \simeq 8 \times 10^{18} \mathrm{~cm}^{-3}$, a charge of $\simeq 30 \mathrm{pC}$ is reproducibly accelerated [9] with an energy distribution of $10 \mathrm{pC}$ in a peak around $210 \mathrm{MeV}$ and a plateau background of $20 \mathrm{pC}$, ranging down to energies of $100 \mathrm{MeV}$. The laser pulse and plasma radiation are blocked by a thin aluminum foil. For electron beam collimation, we used a pair of miniature permanent magnet quadrupole lenses with a field gradient of $\sim 500 \mathrm{~T} / \mathrm{m} \mathrm{[10].} \mathrm{The} 17$ and $15 \mathrm{~mm}$ long lenses are located $\sim 20 \mathrm{~cm}$ after the exit of the accelerator and arranged such that they collimate an energy of $200 \mathrm{MeV}$. Subsequently, the beam propagates through the undulator, which has a period of $\lambda_{u}=5 \mathrm{~mm}, K=0.55$ and a total length of $30 \mathrm{~cm}$ [10]. The emitted radiation is collected by a spherical gold mirror in grazing incidence geometry and spectrally resolved by a 1000 lines $/ \mathrm{mm}$ transmission grating in combination with an X-ray CCD. The undulator and the electron beam spectrum can be measured simultaneously. 


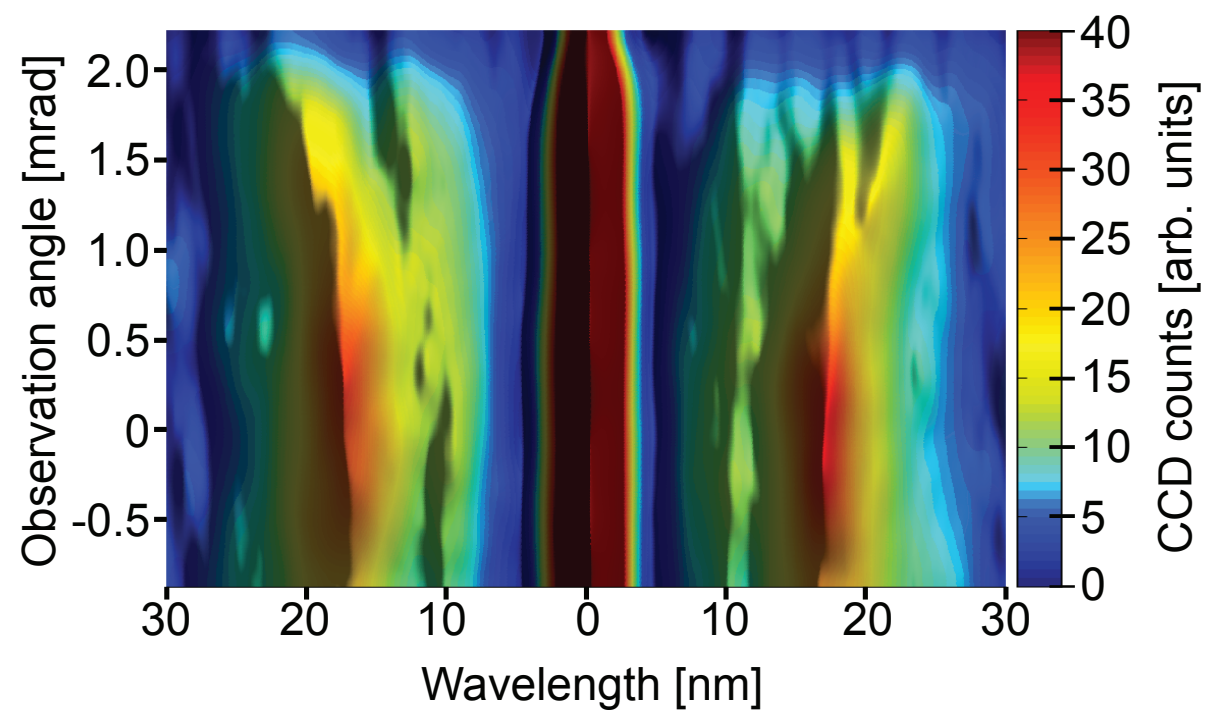

FIGURE 2. Measurement of a single-shot undulator spectrum. The angular distribution of the radiation is spectrally resolved by a transmission grating. The measurement shows the zeroth diffraction (center) and the \pm first diffraction orders (left/right). Both of which consist of a fundamental undulator peak at $17 \mathrm{~nm}$, a second harmonic peak at $9 \mathrm{~nm}$ and a cutoff at $5 \mathrm{~nm}$. The cutoff is caused by the reflectivity of the gold mirror.

\section{EXPERIMENTAL RESULTS}

The undulator radiation shows a fundamental peak at a wavelength of $17 \mathrm{~nm}$ and a second harmonic centered around $9 \mathrm{~nm}$ (figure 2) [11]. The wavelength of the second harmonic is slightly longer than half the wavelength of the fundamental due to the focussing of the gold mirror: the mirror collects the second harmonic radiation, which is peaked off-axis at a longer wavelength (see eq. 1) and horizontally focusses it on axis at the position of the detector. From the measured spectrum, we deduce an on-axis peak intensity of 8,000 photons $/ \mathrm{shot} / \mathrm{mrad}^{2} / 0.1 \%$ bandwidth. For short-wavelength radiation, the pulse duration of the emission is essentially given by that of the electron bunch, which is estimated to be on the order of $10 \mathrm{fs}$. The undulator source is remarkably stable with a detection of spectrally resolved radiation in $70 \%$ of consecutive driver laser shots. The angle-resolved spectra and the dependence of undulator wavelength on the electron energy are in excellent agreement with theory (eq.1).

\section{TOWARD THE TABLE-TOP FEL}

With its ultrashort pulses and small size, the undulator source not only has unique properties itself, but it is a first milestone on the path toward a table-top FEL (TT-FEL). A TT-FEL will be based on a setup similar to that used for the spontaneous undulator 
radiation. The stable operation of our proof-of-principle experiment heavily relies on the use of the magnetic lenses (besides the stable electron beam). The lenses significantly decrease the divergence and the shot-to-shot fluctuation of the electron beam. Control of the beam parameters will be essential for any future undulator- and FEL experiment. However, for a demonstration of an FEL, the requirements on the electron beam in terms of energy spread, charge and emittance are much more stringent and some engineering of the LWFA accelerator is needed. Besides attempts to increase the beam quality by modifying the gas target [12] or the injection mechanism [13], the use of a specifically tailored laser pulse for the plasma conditions have to be used. Our particular future plans are twofold: a spontaneous undulator source emitting radiation with a photon energy of $\sim 20 \mathrm{keV}$ and preferably a repetition rate of $1 \mathrm{kHz}$ and a table-top X-ray FEL (TTXFEL) with photon energies of $\sim 5 \mathrm{keV}$. For the spontaneous source, mainly the electron energy has to be increased (up to $4 \mathrm{GeV}$ ) while keeping the number of accelerated electrons similar to existing experiments $(50 \mathrm{pC})$. The estimated parameters for the driver laser for such a source are pulses with a few Joules in $\sim 25 \mathrm{fs}$, which corresponds to a 300 TW system preferably at a repetition rate of $1 \mathrm{kHz}$. The accelerated charge of an electron beam that could drive the TTXFEL has to be considerably higher $(1 \mathrm{nC})$, whereas the electron energy is less $(2 \mathrm{GeV})$. The laser system that can accelerate such electron beams would have to deliver pulses with several tens of Joules and a pulse duration of $\sim 25 \mathrm{fs}$, which corresponds to a few-PW system.

\section{CONCLUSIONS \& OUTLOOK}

Our laser-driven soft-X-ray undulator source opens the prospect of high-brightness $\mathrm{X}$-ray sources on the university-laboratory scale. The wavelengths can be expanded into the hard-X-ray range by increasing the electron energy. Such a source with its ultrashort pulse duration does not only have unique properties in itself, but can also be considered as a test bed for future table-top FELs. Due to the requirement of even more powerful driver lasers our concept heavily relies on future laser developments, as they are envisaged for example in the ELI program.

\section{ACKNOWLEDGMENTS}

This work has been financially supported by the Deutsche Forschungsgemeinschaft (DFG) through Transregio TR18 and supported by the DFG Cluster-of-Excellence 'Munich-Centre for Advanced Photonics' MAP.

\section{REFERENCES}

1. D. H. Bilderback, P. Elleaume, and E. Weckert, Journal of Physics B: Atomic, Molecular and Optical Physics 38, S773-S797 (2005).

2. C. G. R. Geddes, C. Toth, J. van Tilborg, E. Esarey, C. B. Schroeder, D. Bruhwiler, C. Nieter, J. Cary, and W. P. Leemans, Nature 431, 538-541 (2004). 
3. S. P. D. Mangles, C. D. Murphy, Z. Najmudin, A. G. R. Thomas, J. L. Collier, A. E. Dangor, E. J. Divall, P. S. Foster, J. G. Gallacher, C. J. Hooker, D. A. Jaroszynski, A. J. Langley, W. B. Mori, P. A. Norreys, F. S. Tsung, R. Viskup, B. R. Walton, and K. Krushelnick, Nature 431, 535-538 (2004).

4. J. Faure, Y. Glinec, A. Pukhov, S. Kiselev, S. Gordienko, E. Lefebvre, J. P. Rousseau, F. Burgy, and V. Malka, Nature 431, 541-544 (2004).

5. A. Pukhov, and J. Meyer-ter Vehn, Applied Physics B: Lasers and Optics 74, 355-361 (2002).

6. E. Esarey, C. B. Schroeder, and W. P. Leemans, Reviews of Modern Physics 81, 1229 (2009).

7. F. Gruener, S. Becker, U. Schramm, T. Eichner, M. Fuchs, R. Weingartner, D. Habs, J. Meyer-ter Vehn, M. Geissler, M. Ferrario, L. Serafini, B. van der Geer, H. Backe, W. Lauth, and S. Reiche, Applied Physics B: Lasers and Optics 86, p431 - 435 (2007).

8. K. Nakajima, Nat Phys 4, 92-93 (2008).

9. J. Osterhoff, A. Popp, Z. Major, B. Marx, T. P. Rowlands-Rees, M. Fuchs, M. Geissler, R. Horlein, B. Hidding, S. Becker, E. A. Peralta, U. Schramm, F. Gruner, D. Habs, F. Krausz, S. M. Hooker, and S. Karsch, Physical Review Letters 101, 085002 (2008).

10. T. Eichner, F. Grüner, S. Becker, M. Fuchs, D. Habs, R. Weingartner, U. Schramm, H. Backe, P. Kunz, and W. Lauth, Phys. Rev. ST Accel. Beams 10, 082401 (2007).

11. M. Fuchs, R. Weingartner, A. Popp, Z. Major, S. Becker, J. Osterhoff, I. Cortrie, B. Zeitler, R. Horlein, G. D. Tsakiris, U. Schramm, T. P. Rowlands-Rees, S. M. Hooker, D. Habs, F. Krausz, S. Karsch, and F. Gruner, Nat Phys 5, 826-829 (2009).

12. C. G. R. Geddes, K. Nakamura, G. R. Plateau, C. Toth, E. Cormier-Michel, E. Esarey, C. B. Schroeder, J. R. Cary, and W. P. Leemans, Physical Review Letters 100, 215004 (2008).

13. J. Faure, C. Rechatin, A. Norlin, A. Lifschitz, Y. Glinec, and V. Malka, Nature 444, 737-739 (2006). 